\title{
New Research in Nature Inspired Algorithms for Mobility Management in GSM Networks
}

\author{
Enrique Alba ${ }^{1}$, José García-Nieto ${ }^{1}$, Javid Taheri ${ }^{2}$, and Albert Zomaya ${ }^{2}$ \\ ${ }^{1}$ Dept. de Lenguajes y Ciencias de la Computación, University of Málaga, \\ ETSI Informática, Campus de Teatinos, Málaga - 29071, Spain \\ \{eat, jnieto\}@lcc.uma.es \\ ${ }^{2}$ School of Information Technologies, University of Sydney, \\ Sydney, NSW 2006, Australia \\ \{javidt,zomaya\}@it.usyd.edu.au
}

\begin{abstract}
Mobile Location Management (MLM) is an important and complex telecommunication problem found in mobile cellular GSM networks. Basically, this problem consists in optimizing the number and location of paging cells to find the lowest location management cost. There is a need to develop techniques capable of operating with this complexity and used to solve a wide range of location management scenarios. Nature inspired algorithms are useful in this context since they have proved to be able to manage large combinatorial search spaces efficiently. The aim of this study is to assess the performance of two different nature inspired algorithms when tackling this problem. The first technique is a recent version of Particle Swarm Optimization based on geometric ideas. This approach is customized for the MLM problem by using the concept of Hamming spaces. The second algorithm consists of a combination of the Hopfield Neural Network coupled with a Ball Dropping technique. The location management cost of a network is embedded into the parameters of the Hopfield Neural Network. Both algorithms are evaluated and compared using a series of test instances based on realistic scenarios. The results are very encouraging for current applications, and show that the proposed techniques outperform existing methods in the literature.
\end{abstract}

Keywords: Mobile Location Management, GSM Cellular Networks, Geometric Particle Swarm Optimization, Hopfield Neural Network.

\section{Introduction}

Mobility Management becomes a crucial issue when designing infrastructure for wireless mobile networks. In order to route incoming calls to appropriate mobile terminals, the network must keep track of the location of each mobile terminal. Mobility management requests are often initiated either by a mobile terminal movement (crossing a cell boundary) or by deterioration of the quality of a received signal in a currently allocated channel. Due to the expected increase in the usage of wireless services in the future, the next generation of mobile networks should be able to support a huge number of users and their bandwidth requirements 14 . 
Several strategies for Mobility Management have been used in the literature being the location area (LA) scheme one of the most popular 611]. An analogous strategy is the Reporting Cells (RC) scheme suggested in [3]. In RC, a subset of cells in the network is designated as reporting cells. Each mobile terminal performs a location update only when it enters one of these reporting cells. When a call arrives, the search is confined to the reporting cell the user last reported and the neighboring bounded nonreporting cells. It was shown in 3 that finding an optimal set of reporting cells, such that the location management cost is minimized, is an NP-complete problem. For this reason, bioinspired algorithms have been commonly used to solve this problem 710.

In this work, we use two nature inspired algorithms to assign the reporting cells of a network following the RC scheme. The first algorithm, called Geometric Particle Swarm Optimization (GPSO), is a generalization of the Particle Swarm Optimization for virtually any solution representation, which works according to a geometric framework. The second technique combines a Hopfield Neural Network with a Ball Dropping (HNN+BD) mechanism. Our contributions are both to perform better with respect to existing works and to introduce the GPSO algorithm for solving Telecommunications problems. In addition, these two techniques are experimentally assessed and compared from different points of view such as quality of the solutions, the robustness and design issues.

The remaining of the paper is organized as follows: Section 2 briefly explains the Mobility Management problem. The two algorithms, GPSO and HNN+BD, are described in sections 3 and 4 respectively. After that, Section 5 presents a number of experiments and results that show the applicability of the proposed approaches to this problem. Finally, conclusions are drawn in Section 6 .

\section{The Mobility Management Problem}

Basically, the Mobility (location) Management problem consists in reducing the total cost of managing a mobile cellular network. Two factors take part when calculating the total cost: the updating cost and the paging cost. The updating cost is the portion of the total cost due to location updates performed by roaming mobile terminals in the network. The paging cost is caused by the network during a location inquiry when the network tries to locate a user 1 .

According to the reporting cells scheme, there are two types of cells: reporting cells (RC) and non-reporting cells (nRC). A neighborhood is assigned to each reporting cell, which consists of all $\mathrm{nRC}$ that must also page the user in case of an incoming call. For both RC and $\mathrm{nRC}$, a vicinity factor is calculated representing the maximum number of reporting neighbors for each cell that must page the user (including the cell itself) in case of an incoming call. Obviously, the vicinity factor of each RC is the number of neighbors it has (see Fig. 1).

\footnotetext{
${ }^{1}$ Other costs like the cost of database management to register user's locations or the cost of the wired network (backbone) that connects the base stations to each other were not considered here, since these costs are assumed to be the same for all location management strategies and hence aren't contemplated in comparisons.
} 


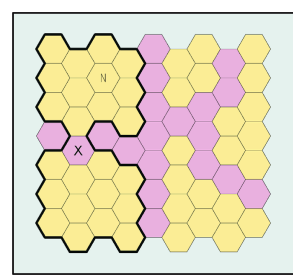

(a)

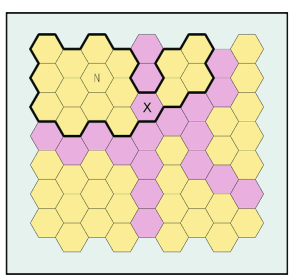

(b)

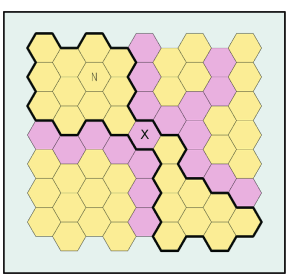

(c)

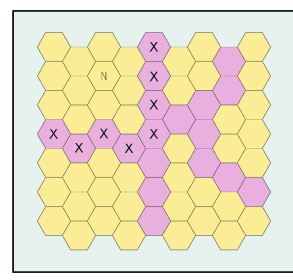

(d)

Fig. 1. Cells marked as ' $N$ ' belong to the neighborhoods of at least three RCs (grey cells). For example, the number of neighbors for cell ' $\mathrm{X}$ ' is 25, 17, and 22 for (a), (b) and (c) respectively ( 25 to consider the worst case). However, if a $\mathrm{nRC}$ belongs to more than two neighborhoods the calculation must be done for all of them, and then, the maximum number is considered as the vicinity factor for this nRC. For example, the $\mathrm{nRC}$ marked as ' $\mathrm{N}$ ' is a part of the neighborhood of all cells marked as ' $\mathrm{X}$ ' in (d).

For $\mathrm{nRC}$, the vicinity factor is calculated based on the fact that each $\mathrm{nRC}$ might be in the neighborhood of more than one $\mathrm{RC}$, the maximum number of paging neighbors that contains such a cell is considered its vicinity factor.

Therefore, to calculate the total cost of the network location management, the general cost function is formulated as:

$$
\text { Cost }=\beta \times \sum_{i \in S} N_{L U}(i)+\sum_{i=0}^{N} N_{P}(i) \times V(i)
$$

where, $N_{L U}(i)$ is the number of location updates for reporting cell number $i$, $N_{P}(i)$ is the number of arrived calls for cell $i, V(i)$ is the vicinity factor for cell $i, S$ is the set of cells defined as reporting cells, and $N$ is the total number of cells in the network. $\beta$ is a constant representing the cost ratio of a location update to a paging transaction in the network (typically $\beta=10$ ). This function is used either as fitness function by the GPSO or energy function by the HNN.

\section{Geometric Particle Swarm Optimization}

The recent Geometric Particle Swarm Optimization (GPSO) [512, enables us to generalize PSO to virtually any solution representation in a natural and straightforward way, extending the search to richer spaces, such as combinatorial ones. This property was demonstrated for the cases of Euclidean, Manhattan and Hamming spaces in the referenced work.

The key issue in this approach consists of using a multi-parental recombination of particles which leads to the generalization of a mask-based crossover operation, proving that it respects four requirements for being a convex combination in a certain space (see [5] for a complete explanation). This way, the mask-based crossover operation substitutes the classical movement in PSO, based on the velocity and position update operations, only suited for continuous spaces.

For Hamming spaces, which is the focus of this work, a three-parent maskbased crossover (3PMBCX) was defined in a straightforward way: 
Definition 1. Given three parents $a, b$ and $c$ in $\{0,1\}^{n}$, generate randomly $a$ crossover mask of length $n$ with symbols from the alphabet $\{a, b, c\}$. Build the offspring o filling each position with the bit from the parent appearing in the crossover mask at the position.

In a convex combination, the weights $w_{a}, w_{b}$ and $w_{c}$ indicate for each position in the crossover mask the probability of having the symbols $a, b$ or $c$.

The pseudocode of the GPSO algorithm for Hamming spaces is illustrated in Algorithm 1, For a given particle $i$, three parents take part in the 3PMBCX operator (line 13): the current position $x_{i}$, the social best position $g_{i}$ and the historical best position found $h_{i}$ (of this particle). The weight values $w_{a}, w_{b}$ and $w_{c}$ indicate for each element in the crossover mask the probability of having values from the parents $x_{i}, g_{i}$ or $h_{i}$ respectively. A constriction of the geometric crossover forces $w_{a}, w_{b}$ and $w_{c}$ to be non-negative and add up to one.

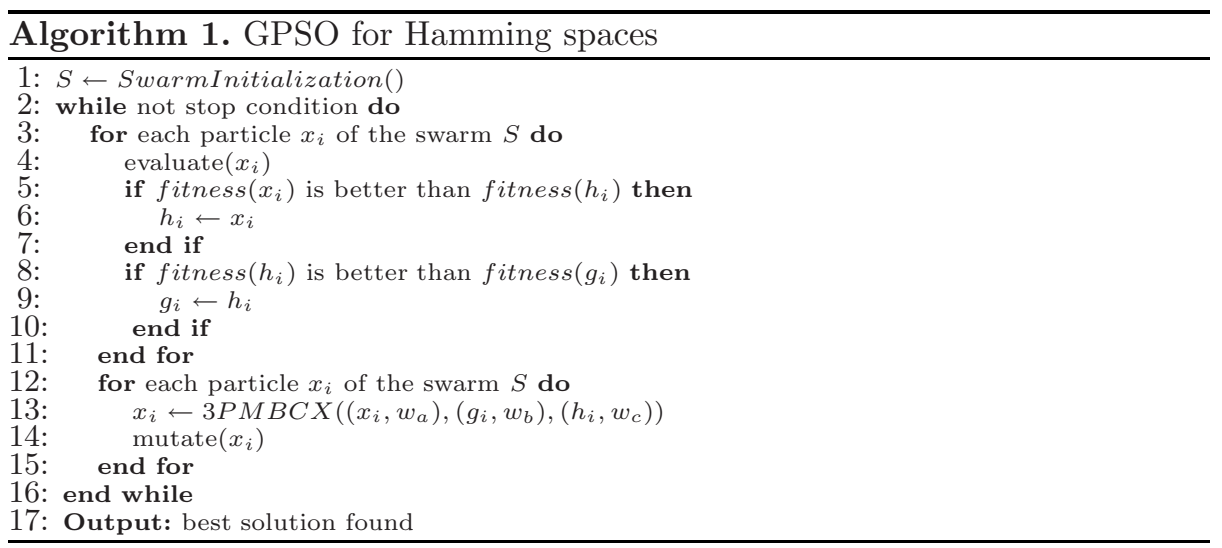

Since the GPSO for Mobility Management was developed for Hamming space, each particle $i$ of the swarm consists of a binary vector $x_{i}=\left(x_{i 1}, x_{i 2}, \ldots, x_{i n}\right)$ representing a reporting cell configuration, where each element $x_{i j}$ represents a cell of the network; $x_{i j}$ can have a value of either "0", representing a nRC, or " 1 ", representing a RC. For example, in an $6 \times 6$ network, the particle position will have a length $(n)$ of 36 .

\section{Hopfield Neural Network with Ball Dropping}

In this approach, the Ball Dropping technique is used as the backbone of the algorithm that employs the HNN as its optimizer, and is inspired by the natural behavior of individual balls when they are dropped onto a non-even plate (a plate with troughs and crests). As can be expected, the balls will spontaneously move to the concave areas of the plate, and in a natural process, find the minimum of the plate. A predefined number of balls are dropped onto several random positions on the plate, which is equivalent to the random addition of a predefined number of paging cells to the current paging cell configuration of the network. 
As a result, after dropping a number of balls on the plate the energy value of the network increases suddenly and the HNN optimizer tries to reduce it by moving the balls around. The following procedure summarizes the basic form of this algorithm.

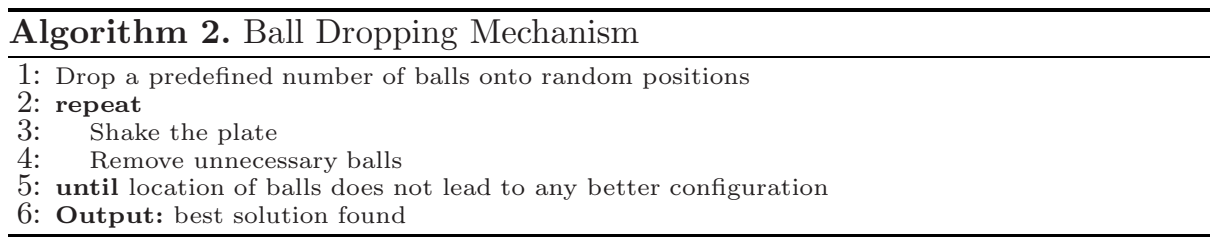

In relation to Equation [1, the state vector of the $\mathrm{HNN}$, ' $\mathrm{X}$ ', is considered to have two different components for location updates and call arrival as follows:

$$
X=\left[\begin{array}{llllllll}
x_{0} & x_{1} & \wedge & x_{N-1} & x_{N} & x_{N+1} & \wedge & x_{2 N-1}
\end{array}\right]^{T}
$$

where $x_{0}$ to $x_{N-1}$ is the location updates part, $x_{N}$ to $x_{2 N-1}$ is the call arrival part and ' $\mathrm{N}$ ' is the total number of cells in the network. This HNN model is designed to represents a RC configuration network, and then, tries to modify its $\mathrm{RCs}$ in order to reduce the total cost gradually. To summarize this explanation, we refer the reader to [8] where other aspects like generating a initial solution generation, definition of function to modify the state vector and reduction of the number of variations are given completely.

\section{Simulation Results}

In this section we present the experiments conducted to evaluate and compare the proposed GPSO and HNN+BD. We firstly give some details of the test network instances used. The experiments with both algorithms are presented and analyzed afterwards. We have made 10 independent runs for each algorithm and instance. Comparisons are made from different points of view such as the performance, robustness, quality of solutions and even design issues concerning the two algorithms. Finally, comparisons with other optimizers found in the literature are encouraging since our algorithms obtain competitive solutions which even beat traditional metaheuristic techniques in the previous state of the art.

\subsection{Test GSM Network Instances}

In almost all of the previous research in the literature, the cell attributes of the network are generated randomly. In general, two independent attributes for each cell are considered: the number of call arrivals $(N P)$ and the number of location updates $(N L U)$, which are set at random according to a normal distribution. However, these numbers are highly correlated in real world scenarios. Therefore, in this work, a more robust and realistic approach is used to seed the initial solutions, and consequently, the network attributes of each cell [9]. This makes the configuration of the solutions obtained in this work to be more realistic. 
Therefore, a benchmark of twelve different instances were generated here to be used for testing GPSO and HNN+BD. The numeric values shaping the test networks configurations are given in tables below 2 for future reproduction of our results.

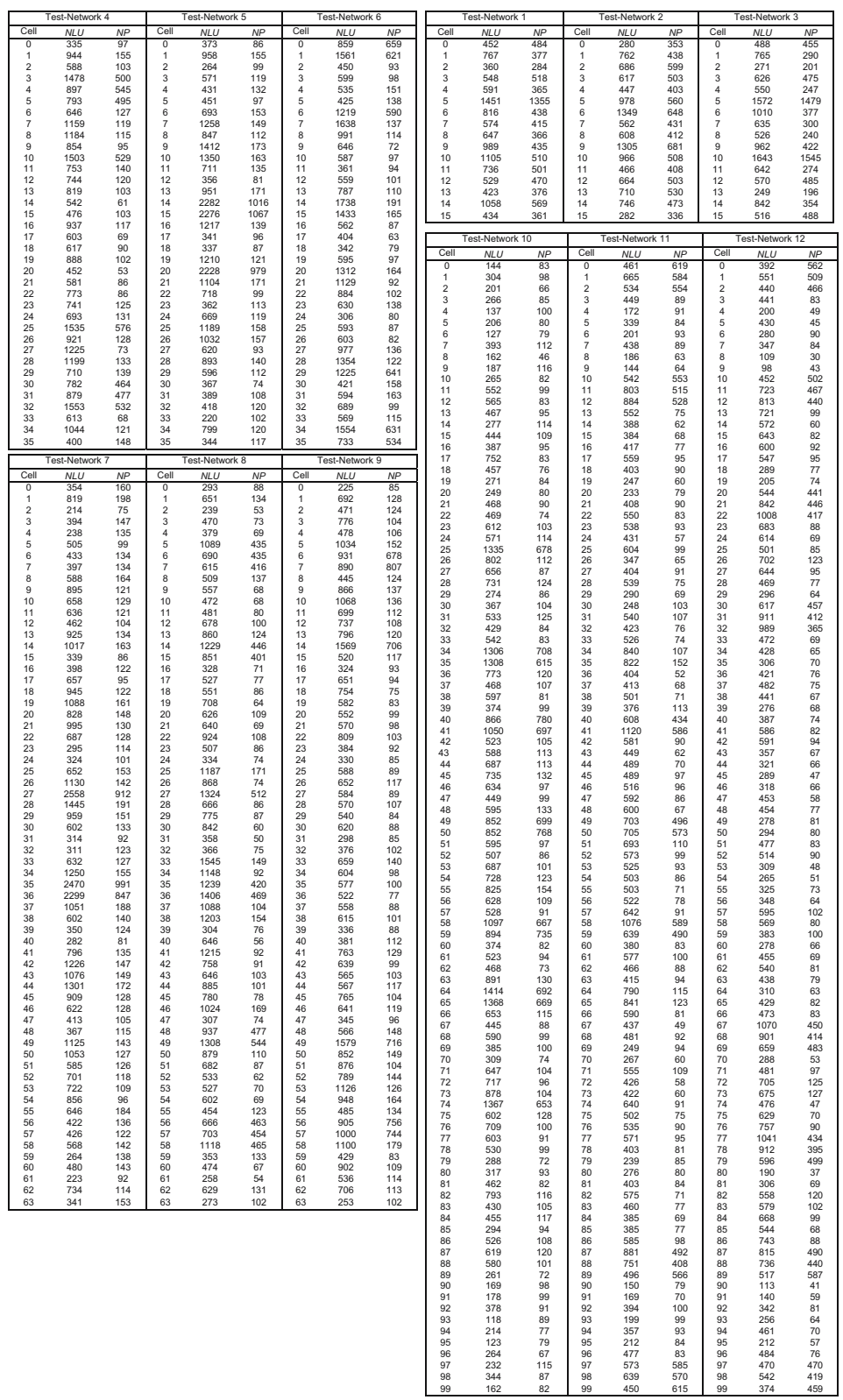

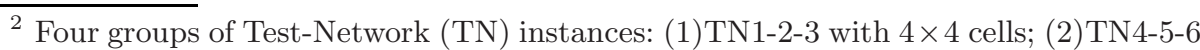
with $6 \times 6$ cells; (3)TN7-8-9 with $8 \times 8$ cells; (4)TN10-11-12 with $10 \times 10$ cells. TN files are available in URL http://oplink.lcc.uma.es/problems/mmp.html.
} 


\subsection{Experimental Results}

We have conducted different experiments with several configurations of GPSO and HNN+BD depending on the test network used. Since the two algorithms perform quite different operations, we have set the parameters (Table 11) after preliminary executions of the two algorithms (with each instance) where the computational effort in terms of time and number of evaluations was balanced.

Table 1. Parameter settings for $\mathrm{HNN}+\mathrm{BD}$ and GPSO. The columns indicate: the number of dropping balls (N.DroppBalls) and the number of trials (N.Trials) for HNN+BD. For GPSO are reported: the number of particles (N.Particles), the crossover probability $\left(P_{\text {cross }}\right)$, the mutation probability $\left(P_{m u t}\right)$ and the weighted values $\left(w_{a}, w_{b}\right.$ and $\left.w_{c}\right)$.

\begin{tabular}{|c|c|c|c|c|c|c|}
\hline Test Network & \multicolumn{2}{|c|}{ HNN+BD } & \multicolumn{4}{c|}{ GPSO } \\
\hline Dim. & N.DroppBalls & N.Trials & N.Particles & $P_{\text {cross }}$ & $P_{\text {mut }}$ & $w_{a}+w_{b}+w_{c}$ \\
\hline$(4 \times 4)$ & 7 & 3 & 20 & & & \\
$(6 \times 6)$ & 10 & 5 & 50 & & & \\
$(8 \times 8)$ & 15 & 5 & 100 & & 0.1 & $0.33+0.33+0.33$ \\
$(10 \times 10)$ & 15 & 5 & 120 & & & \\
\hline
\end{tabular}

After the initial experimentation, several results were obtained; they are shown in Table 2. The first column contains the number and dimension (in parenthesis) of each test network. Three values are presented for each evaluated algorithm: the best cost (out of 10 runs), the average cost (Aver.) of all the solutions, and the deviation ( Dev.) percentage from the best cost.

As it can be seen from the results, the two algorithms have similar performance in almost all of the instances, although there are a few differences for the large test networks. For example, GPSO obtains better solutions in Test-Network 7 and 10, while, HNN+BD obtains a better solution in Test-Network 11. In addition, it can be noticed that the deviation percentage from the best cost is generally lower in GPSO than in $\mathrm{HNN}+\mathrm{BD}$, specially for the smaller test networks. This behavior leads us to believe that the GPSO approach is more robust than $\mathrm{HNN}+\mathrm{BD}$, but just slightly.

Table 2. Results for Test Networks obtained by HNN+BD and GPSO

\begin{tabular}{|c|c|c|c|c|c|c|}
\hline Test Network & \multicolumn{3}{|c|}{ HNN+BD } & \multicolumn{3}{c|}{ GPSO } \\
\hline No.(Dim.) & Best & Aver. & Dev. & Best & Aver. & Dev. \\
\hline $1(4 \times 4)$ & 98,535 & 98,627 & $0.09 \%$ & 98,535 & 98,535 & $0.00 \%$ \\
$2(4 \times 4)$ & 97,156 & 97,655 & $0.51 \%$ & 97,156 & 97,156 & $0.00 \%$ \\
$3(4 \times 4)$ & 95,038 & 95,751 & $0.75 \%$ & 95,038 & 95,038 & $0.00 \%$ \\
\hline $4(6 \times 6)$ & 173,701 & 174,690 & $0.56 \%$ & 173,701 & 174,090 & $0.22 \%$ \\
$5(6 \times 6)$ & 182,331 & 182,430 & $0.05 \%$ & 182,331 & 182,331 & $0.00 \%$ \\
$6(6 \times 6)$ & 174,519 & 176,050 & $0.87 \%$ & 174,519 & 175,080 & $0.32 \%$ \\
\hline $7(8 \times 8)$ & 308,929 & 311,351 & $0.78 \%$ & 308,401 & 310,062 & $0.53 \%$ \\
$8(8 \times 8)$ & 287,149 & 287,149 & $0.00 \%$ & 287,149 & 287,805 & $0.22 \%$ \\
$9(8 \times 8)$ & 264,204 & 264,695 & $0.18 \%$ & 264,204 & 264,475 & $0.10 \%$ \\
\hline $10(10 \times 10)$ & 386,351 & 387,820 & $0.38 \%$ & 385,972 & 387,825 & $0.48 \%$ \\
$11(10 \times 10)$ & 358,167 & 359,036 & $0.24 \%$ & 359,191 & 359,928 & $0.20 \%$ \\
$12(10 \times 10)$ & 370,868 & 374,205 & $0.89 \%$ & 370,868 & 373,722 & $0.76 \%$ \\
\hline
\end{tabular}


Another obvious difference between HNN+BD and GPSO lies in the behavior of each algorithm. This can be observed in Fig. 2. where we show a graphical representation of algorithm runs for the different evaluated networks. Each graph, corresponding to one of the twelve test networks, plots a representative trace of the execution of each algorithm tracking the best solution obtained versus the number of iterations. On the one hand, GPSO shows a typical behavior in evolutionary metaheuristics, that is, it tends to converge from the solutions in the initial population to an optimal reporting cell arrangement. Graphically, the GPSO operation is represented by a monotonous decreasing (minimization) curve. On the other hand, HNN+BD carries out a different searching strategy, as from the initialization, it provokes frequent shaking scenarios in the population with the purpose of gradually diversifying and intensifying the search. These "shakes" are carried out by means of the Ball Dropping technique (Section 4) when no improvement in the overall condition of the network is detected, so the frequency of this operation is variable.
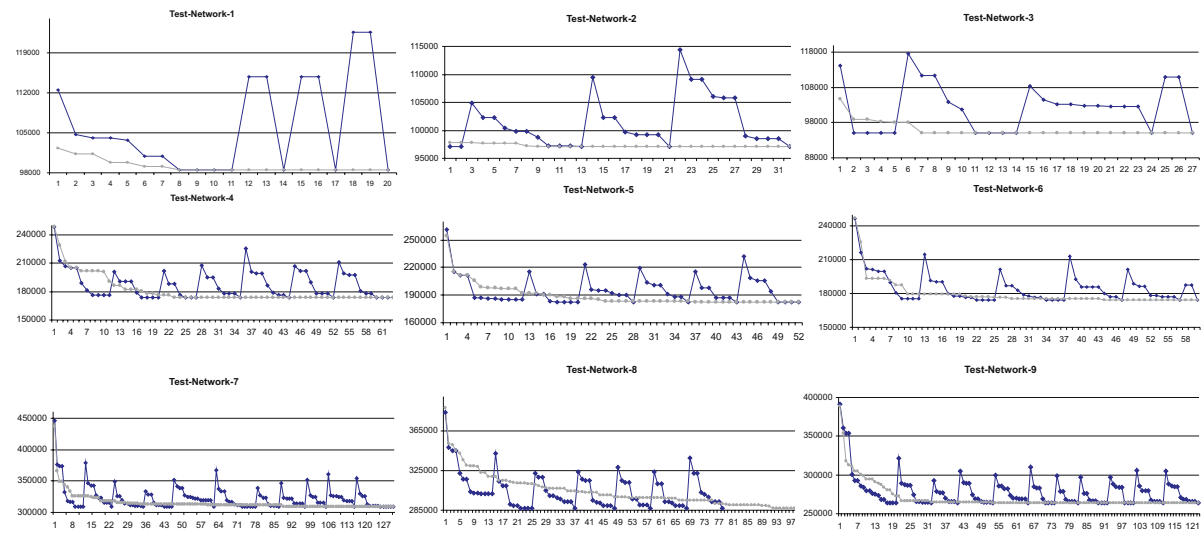

Test-Network-1

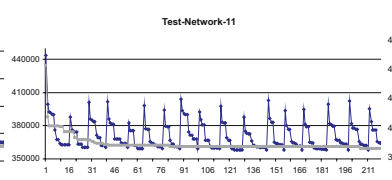

Test-Network12
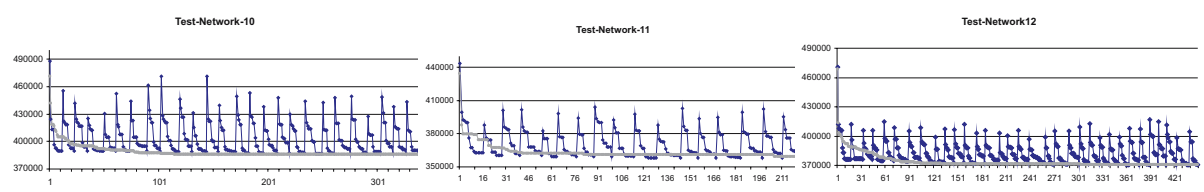

Fig. 2. Cost values level ( $\mathrm{Y}$ axis) versus iterations ( $\mathrm{X}$ axis) of all the test networks. Each graphic plots the energy level obtained, we track the evolution of the HNN+BD algorithm (black line with peaks and valleys), and the fitness level in the evolution of the GPSO algorithm (concave grey curve).

Evidently, as Fig. 2 shows, the number of drops in larger test networks is higher than in smaller ones, since the number of iterations required here to converge is also higher. Graphically, this behavior produces intermittent peaks and valleys in the evolution line. 
From the point of view of the quality of solutions, as expected, optimal reporting cell configurations for all test networks split the network into smaller sub-networks by clustering the full area. This property can be seen in the large instances in a much clearer way than in the short ones (Fig. 3).

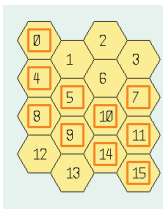

Test Network 2 Best Cost: 97,156

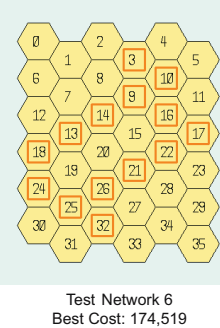

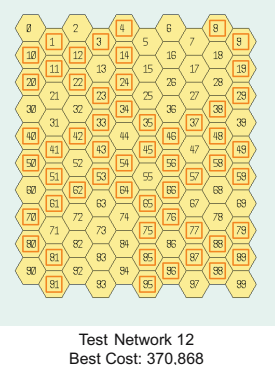

Fig. 3. Paging Cells (with squares) configurations obtained as solutions by the two algorithms (the same solutions) in Test Network 2, Test Network 6, Test Network 8 and Test Network 12. Neighborhood area clusters are easily visible in larger instances. All the legends show the Best Cost found by both algorithms.

\subsection{Comparison with Other Optimizers}

To the best of our knowledge a Genetic Algorithm (GA) is the only algorithm that can be compared against in this work. The modeling of the problem, the quality of the initial population, and the number of iterations are the main design issues that can affect the performance of the GA. When comparing the proposed approaches with a GA implementation given in [7, one can observe two advantages in terms of convergence and quality of solution in our two new approaches.

Despite the general good behavior of the GA, our two approaches generate a better solution when solving the Test-Network-2 $(6 \times 6$ instance provided in [7) in additional experiments. The energy value obtained by the GA is 229,556 with a total of 26 paging cells in the network, while, the cost obtained by $\mathrm{HNN}+\mathrm{BD}$ in this work is 211,278 with 24 paging cells, and the GPSO obtained a cost of 214,313 with 23 paging cells. With respect to HNN+BD, a reasonable explanation for this difference could be due to the setup parameters used for the GA in [7]. However, our GPSO uses a similar setup parameters compared to the GA, providing a better solution with a smaller number of paging cells.

\section{Conclusions}

This paper addresses the use of two nature inspired approaches to solve the Mobile Location Management problem found in telecommunications: a new binary Particle Swarm Optimization algorithm called GPSO, and an algorithm based on a Hopfield Neural Network hybridized with the Balls Dropping Technique. 
The problem is described and tackled following the Reporting Cells Scheme. In addition, the design and operation of HNN+BD and GPSO are discussed. Twelve test networks of different dimensions, generated following realistic scenarios of mobile networks, were for the first time used in this work. In addition, a comparison of the algorithms is carried out focusing on the performance, robustness, and design issues.

In conclusion, simulation results are very encouraging and show that the proposed algorithms outperform existing methods. Both approaches prove themselves as very powerful optimizers providing fast and good quality solutions.

This work has been carried out as a continuation of previous works where metaheuristics techniques were applied to solve the Mobile Location Management problem. For further work, we are interested in evaluating new test networks under different conditions of topology and dimension. In addition, new experiments will be carried out using different location area schemes.

\section{References}

1. Agrawal, D.P., Zeng, Q.-A.: Introduction to Wireless and Mobile Systems. Thomson Brooks/Cole Inc. (2003)

2. Alba, E., García-Nieto, J., Jourdan, L., Talbi, E.-G.: Gene Selection in Cancer Classification using PSO/SVM and GA/SVM Hybrid Algorithms. In: IEEE Congress on Evolutionary Computation CEC-2007, Singapore (September 2007)

3. Bar-Noy, A., Kessler, I.: Tracking mobile users in wireless communication networks. In: INFOCOM (3), pp. 1232-1239 (1993)

4. Lin, Y.-B., Chlamatac, I.: Wireless and Mobile Network Architecture. John Wiley and Sons, Chichester (2001)

5. Moraglio, A., Di Chio, C., Poli, R.: Geometric Particle Swarm Optimization. In: Ebner, M., O’Neill, M., Ekárt, A., Vanneschi, L., Esparcia-Alcázar, A.I. (eds.) EuroGP 2007. LNCS, vol. 4445, Springer, Heidelberg (2007)

6. Subrata, R., Zomaya, A.Y.: Location management in mobile computing. In: ACS/IEEE International Conference on Computer Systems and Applications, pp. 287-289 (2001)

7. Subrata, R., Zomaya, A.Y.: A comparison of three artificial life techniques for reporting cell planning in mobile computing. IEEE Trans. Parallel Distrib. Syst. 14(2), 142-153 (2003)

8. Taheri, J., Zomaya, A.Y.: The use of a hopfield neural network in solving the mobility management problem. In: IEEE/ACS International Conference on Pervasive Services, ICPS 2004, pp. 141-150 (July 2004)

9. Taheri, J., Zomaya, A.Y.: Realistic simulations for studying mobility management problems. Int. Journal of Wireless and Mobile Computing 1(8) (2005)

10. Taheri, J., Zomaya, A.Y.: A genetic algorithm for finding optimal location area configurations for mobility management. In: LCN 2005: Proceedings of the The IEEE Conference on Local Computer Networks 30th Anniversary, Washington, DC, USA, pp. 568-577. IEEE Computer Society, Los Alamitos (2005)

11. Wu, H.-K., Jin, M.-H., Horng, J.-T., Ke, C.-Y.: Personal paging area design based on mobile's moving behaviors. In: Twentieth Annual Joint Conference of the IEEE Computer and Communications Societies, INFOCOM 2001, vol. 1, pp. 21-30 (2001) 